\title{
Observing $\gamma$ Doradus Stars with BRITE - an Outlook
}

\author{
M. Gruberbauer ${ }^{1}$, R. Neuteufel ${ }^{1}$, W. W. Weiss ${ }^{1}$ \\ ${ }^{1}$ Institut für Astronomie, Türkenschanzstrasse 17, 1180 Vienna, Austria
}

\section{Abstract}

We discuss the advantages and challenges of observing $\gamma$ Doradus stars with the BRITE-Constellation satellite network and how using these instruments can improve our understanding of these objects.

\section{Introduction}

$\gamma$ Doradus (hereafter $\gamma$ Dor) stars are a relatively new class of pulsating stars and have been defined in Kaye et al. (1999). These stars are of spectral type A7 to F5 (IV-V) and are, as such, crossing the red edge of the classical instability strip in the HR diagram, close to the much better studied class of $\delta$ Scuti stars. The pulsations are assumed to be due to high-order, low-degree g-modes, which results in periods ranging from about 8 to 80 hours. Their g-mode nature is what makes $\gamma$ Dor stars very interesting asteroseismic targets, since these modes probe the interiors of stars. While the driving mechanism, attributed to the blocking of radiative energy transport by convection at the base of the convective envelope, is not yet completely understood, models are already able to reproduce basic characteristics of $\gamma$ Dor stars (Dupret et al. 2006). Since their initial discovery, the number of known $\gamma$ Dor pulsators has increased and is now probably close to about 100 stars.

Previous observations show moderate amplitudes of light variation, clustering at about $0.04 \mathrm{mag}$. However, data from current satellite missions like MOST (Microvariability and Oscillation of STars, Walker et al. 2003) yield evidence for much lower amplitudes (e.g. King et al., in preparation) which are hard to detect with ground-based observations. Thus, with current space missions and the advent of even more advanced instruments like COROT (Baglin et al. 2002), a dramatic boost in $\gamma$ Dor discoveries can be expected.

Aside from "normal" $\gamma$ Dor stars also four hybrid pulsators, showing also characteristic frequencies of $\delta$ Scuti stars, have been found as of yet. Two of these discoveries have been made from space (Rowe et al. 2006, King et al. 2006). These are especially interesting, since the presence of $g$ - and p-modes 
allows to probe not only the deep interior but also the outer envelope of the star. An overview of the state of asteroseismology of $\gamma$ Dor stars can be found in Handler (2005).

BRITE (BRIght Target Explorer)-Constellation (hereafter BRITE-C) is a network of nanosatellite pairs designed for asteroseismic observations of the brightest stars $\left(m_{\mathrm{V}}<6\right)$. The satellites will operate in low-Earth orbit (LEO) and as a network, they will be able to observe stars over very long time bases in the order of weeks or months, depending on the position of the target. Each pair will be able to deliver colour information, since each of its satellites is equipped with a different filter. For more information on BRITE-C we refer to other papers in these proceedings.

\section{$\gamma$ Dor stars and Space Missions}

For every type of pulsating stars, mode identification is the primary goal of frequency analysis and in most cases a necessary step for fitting model frequencies to test pulsation theory. Alas, $\gamma$ Dor stars have several properties which make mode identification a very delicate problem. Since $\gamma$ Dor stars have periods which are of the same order as their rotation periods, the impact of rotational effects on the frequency spectrum has to be considered. Even more fundamental, the time-scale of their oscillations can lead to misinterpretation of other types of variability (e.g. binarity, rotational modulation) or instrumental trends as g-mode pulsation. It is therefore very important that each newly proposed member of the $\gamma$ Dor class is unambiguously identified as a pulsating star. For white light photometry as in the case of MOST or the SISMO field of COROT (for a COROT-specific summary on $\gamma$ Dor observations see Mathias et al. 2006), additional colour information or spectroscopy should be taken into consideration, unless the frequency spectrum is dense enough to rule out any other source of variability than stellar oscillations.

\section{Advantages of Observing $\gamma$ Dor Stars with BRITE-C}

As stated in the introduction, BRITE-C is planned to be composed of nanosatellites with different optical filters. Consequently, it will be the only space mission designed for asteroseismology which is able to deliver well-defined colour information. In case of serendipitous discoveries of new $\gamma$ Dor stars, which could not be identified as variable from ground, this will be a very useful tool to asses the nature of their frequencies. Also, the phase differences and amplitude ratios between the different filters will help to put constraints on the mode identification. 
BRITE-C is designed to look at stars over a very long time-base and without daily gaps and hence avoiding $1 d^{-1}$ aliasing, which is common to singlesite observations from ground. The merit is a much cleaner window function, compared to ground-based observations.

Long time-bases are very important for increasing the frequency resolution which is proportional to $1 / \Delta T$, where $\Delta T$ is the length of the time series. The same proportionality also applies to the frequency uncertainty. Thus, longer time-bases deliver smaller upper limits for the error bars of the actual frequency values (Kallinger et al. 2007). Both, the clean window function and the increase in frequency resolution are needed in order for the dense but narrow frequency spectrum of multi-mode $\gamma$ Dor stars to be clearly resolved. Eventually, also the precision of about $1 \mathrm{mmag}$ per data point, which the designers of BRITE-C are aiming for, is well suited for $\gamma$ Dor stars.

\section{Challenges}

The biggest challenge when employing satellites for highly precise time-series measurements is the stability of the instruments. Satellites in a LEO are subject to scattered earthshine, which produces artificial signal at the satellite's orbital frequency (and harmonics). Additionally, long-term trends and daily modulation of the earthshine intensity, produced by albedo changes due to the Earth's rotation, can show up in the low-frequency part of the spectrum (see Reegen et al. 2006 for a discussion of these effects for the case of the MOST satellite). Constant monitoring and correction for these effects is very important, especially for slowly pulsating stars, whose periods are in the order of several orbit periods. Consequently, we propose that several comparison stars should be observed together with programme stars. This condition will be fulfilled due to the large field of view of 24 degrees where typically 4 bright stars can be observed simultaneously. Having multiple satellites observing the same targets will also be beneficial, due to the fact that purely instrumental trends will have different characteristics for each instrument.

\section{Observing strategy for $\gamma$ Dor stars}

The BRITE-C satellites will most likely not be suitable for extensive variability surveys, since the faintest stars BRITE-C will be able to observe with the required minimum accuracy are at $\mathrm{m}_{\mathrm{V}} \sim 6$. Also, the stars should not lie in crowded fields to ensure that overlapping point spread functions (PSF) of other stars do not introduce parasitic signal. A list of proposed candidates is shown in Table 1. Also, the search for hybrid pulsators amongst these $\gamma$ Dor 


\begin{tabular}{c|c|l|l|l} 
HD & sp. t. & V & reference & comments \\
\hline 218396 & A5V & 5.964 & Rodriguez et al. 2006 & multi-mode $\gamma$ Dor \\
206043 & F2V & 5.783 & Henry et al. 2001 & multi-mode $\gamma$ Dor \\
147787 & F4IV & 5.27 & de Cat et al. 2006 & SB, $\gamma$ Dor cand. \\
166114 & F2V & 5.858 & Handler 1999 & $\gamma$ Dor cand. \\
153580 & F6V & 5.285 & Baade \& Kjeldsen 1997 & B, $\gamma$ Dor cand.
\end{tabular}

Table 1: Promising BRITE-C $\gamma$ Dor targets; SB : spectroscopic binary; B : star in binary system

stars/candidates or targets of the BRITE-C $\delta$ Scuti-programme should be pursued. Ground-based spectroscopy and Adaptive Optics imaging should further help to optimize the target selection.

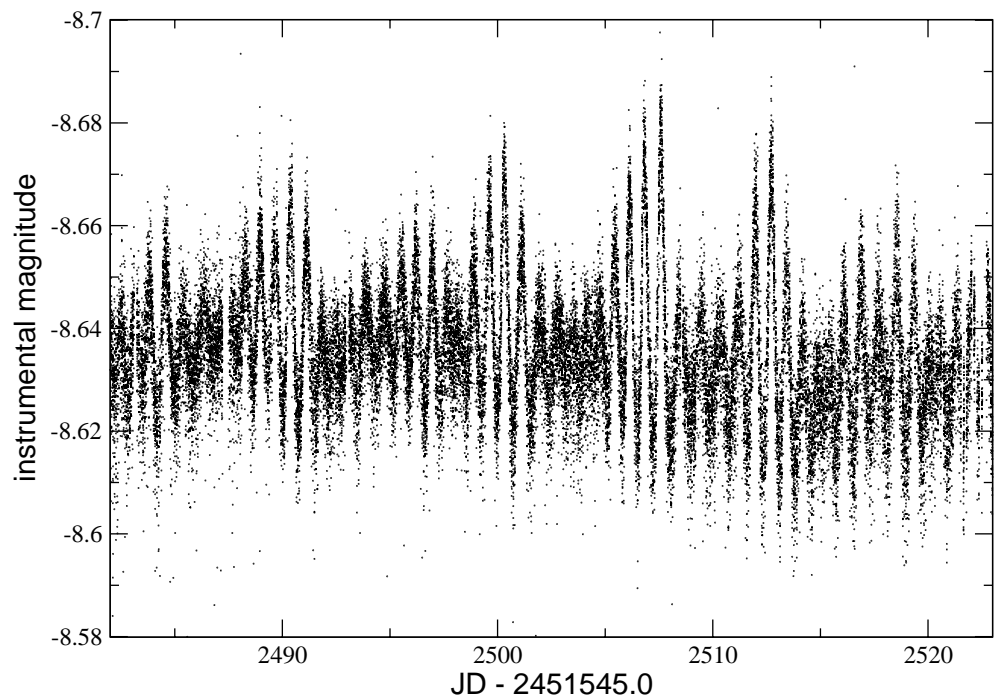

Figure 1: The time series of a recently discovered $\gamma$ Dor star, as observed by the MOST satellite. BRITE-C will be able to deliver similarly exciting results due to the long time-bases and colour information.

\section{Conclusions}

In order to progress with the study of $\gamma$ Dor stars, to improve and test theory (e.g. modelling of eigenfrequencies, driving mechanism, rotational effects) unambiguous detections with optimized instruments are needed. Furthermore, 
it is crucial that the frequency spectra of multi-mode $\gamma$ Dor stars are better understood BRITE-C will be an ideal contributor in this field by delivering data sets of excellent quality. The long time-bases will help to resolve the frequencies in which the most complex $\gamma$ Dor stars pulsate, while the colour information, which no other current or soon-to-be-launched space mission except for BRITE$\mathrm{C}$ is able to deliver, can be used for mode identification. Figure 1 shows a light curve of a $\gamma$ Dor star, recently discovered by the MOST satellite (King et al., in preparation), with a beating signature clearly hinting at multi-mode pulsation. We hope that it is also an outlook on what can be achieved with BRITE-C.

Acknowledgments. The authors would like to thank Gerald Handler for his contribution to the discussion. WWW is supported by the Austrian Science Promotion Agency (FFG - MOST). MG, RN and WWW are supported by the Austrian Science Funds (FWF - P17580).

\section{References}

Baade, D., Kjeldsen, H. 1997, A\&A, 323, 429

Baglin, A., Auvergne, M., Barge, P., et al. 2002, ESA-SP, 485, 17

de Cat, P., Eyer, L., Cuypers, J., et al. 2006, A\&A, 449, 281

Dupret, M.-A., Grigahcène, A., Garrido, R., et al. 2006, MmSAI, 77, 366

Handler, G. 2005, JApA, 26, 24

Handler, G. 1999, MNRAS, 309, 19

Henry, G.W., Fekel, F.C., Kaye, A.B., et al. 2001, AJ, 122, 3383

Kallinger, T., Reegen, P., Weiss, W.W. 2007, A\&A, submitted

Kaye, A.B., Handler, G., Krisciunas, K., et al. 1999, PASP, 111, 840

King, H., Matthews, J.M., Rowe, J.F., et al. 2006, CoAst, 148, 28

Mathias, P., Matar, E., Jankov, S., et al. 2006, MmSAI, 77, 470

Reegen, P., Kallinger, T., Frast, D., et al. 2006, MNRAS, 367, 1417

Rodriguez, E., Costa, V., Zhou, A.-Y., et al. 2006, A\&A, 456, 261

Rowe, J.F., Matthews, J.M., Cameron, C., et al. 2006, CoAst, 148, 34

Walker, G., Matthews, J., Kuschnig, R., et al. 2003, PASP, 115, 1023 